\title{
Long intergenic non-protein coding RNA 152 promotes multiple myeloma progression by negatively regulating microRNA-497
}

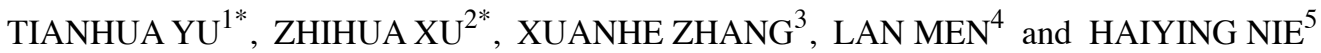 \\ Departments of ${ }^{1}$ Blood Transfusion and ${ }^{2}$ The 3rd Neurology, China-Japan Union Hospital \\ of Jilin University, Changchun, Jilin 130033; ${ }^{3}$ College of Agricultural Science, Shihezi University, \\ Shihezi, Xinjiang 832003; Departments of ${ }^{4}$ Gastrointestinal Medicine and ${ }^{5}$ Vascular Surgery, \\ China-Japan Union Hospital of Jilin University, Changchun, Jilin 130033, P.R. China
}

Received February 19, 2018; Accepted September 13, 2018

DOI: $10.3892 /$ or.2018.6721

\begin{abstract}
Long intergenic non-protein coding RNA 152 (LINC00152, also known as cytoskeleton regulator RNA) is reportedly involved in the development and progression of various types of human malignancy. However, the functional role of LINC00152 in multiple myeloma (MM) and the underlying molecular mechanisms have remained elusive. The present study aimed to investigate the role of LINC00152 in the genesis of MM and the potential molecular mechanisms. It was identified that the expression of LINC00152 was significantly upregulated in plasma cells from patient with MM vs. healthy subjects, and that a high expression LINC00152 was correlated with a shorter оyerall survival in patients with MM. Functional assays demonstrated that knockdown of LINC00152 by transfecting MM cells with LINC00152-specific short hairpin RNA expression plasmids significantly inhibited cell proliferation by inducing cell cycle arrest at the $\mathrm{G}_{0} / \mathrm{G}_{1}$ phase. Furthermore, knockdown of LINC00152 promoted caspase-3/9 activity and apoptosis in MM cells. In addition, knockdown of LINC00152 significantly attenuated tumor growth in mice bearing a myeloma xenograft. A luciferase reporter assay indicated that microRNA (miR)-497 is a direct target of UNC00152, and its expression levels were inversely correlated with those of LINC00152 in MM tissues. Furthermore, repression of miR-497 partly abrogated the inhibitory effects of LINC00152 knockdown on MM
\end{abstract}

Correspondence to: Dr Lan Men, Department of Gastrointestinal Medicine, China-Japan Union Hospital of Jilin University, 126 Xiantai Street, Changchun, Jilin 130033, P.R. China

E-mail: 651172749@qq.com

Dr Haiying Nie, Department of Vascular Surgery, China-Japan Union Hospital of Jilin University, 126 Xiantai Street, Changchun, Jilin 130033, P.R. China

E-mail: niehaiying1818@tom.com

*Contributed equally

Key words: long intergenic non-coding RNA 00152, multiple myeloma, microRNA-497, proliferation, apoptosis cells. Collectively, the results indicated that LINC00152 has an oncogenic effect in MM by targeting miR-497, and may be a novel diagnostic marker and therapeutic target for MM.

\section{Introductio}

Multiple myeloma (MM) is the second most common type of hematologic cancer in the world, characterized by the clonal proliferation of malignant plasma cells, osteolytic bone destruction and pathological fractures $(1,2)$. Although significant progress has been made in MM-associated research, the complex biological and core molecular mechanisms that are involved in the pathogenesis of MM have remained to be fully elucidated (3) Therefore, understanding the molecular events involved in the initiation and progression of MM and identifying novel therapies to treat MM remain a critical, albeit unmet goal.

Long non-coding RNAs (lncRNAs) are a class of transcripts of $>200$ nucleotides in length, which have no protein coding capability (4). Accumulating evidence has revealed that lncRNAs function as master regulators of gene expression at multiple levels, including transcriptional, post-transcriptional and epigenetic modulation $(5,6)$. Several studies have indicated that dysregulated lncRNAs have a crucial role in various biological processes, including cell apoptosis, proliferation and differentiation, by serving as 'micro (mi)RNA sponges' (7). Furthermore, the dysregulated expression of lncRNAs has been indicated to be involved in the development and progression of various human cancer types, and these molecules are considered to be promising biomarkers and therapeutic agents for cancer $(8,9)$. Numerous IncRNAs that have been implicated in MM progression and development by functioning as tumor suppressors or oncogenes $(10,11)$. Consequently, understanding the underlying mechanism and biological functions of lncRNAs in MM may provide new approaches for its treatment.

Long intergenic non-protein coding RNA 152 (LINC00152), also known as cytoskeleton regulator RNA, which is located on chromosome 2p11.2 (12), has been reported to be upregulated in colorectal cancer (13-15), breast cancer (16), lung cancer (17), tongue squamous cell carcinoma (18), gallbladder cancer (19) and renal cell carcinoma (20). These results emphasize the 
importance of the LINC00152 gene as an oncogene. However, the expression patterns and biological functions of LINC00152 in the genesis of $\mathrm{MM}$, and the underlying mechanisms remain elusive. Therefore, LINC00152 expression was measured in MM tissues and cell lines, and a series of in vitro and in vivo experiments was performed to determine the biological functions and a possible molecular basis of LINC00152 in MM.

\section{Materials and methods}

Human tissue samples and cell lines. Plasma cell samples from $40 \mathrm{MM}$ patients (mean age, $62.5 \pm 4.2$ years; age range, 51.3-76.8 years; 22 males and 18 females) and from 40 healthy controls (mean age, 58.3.5 \pm 3.5 years; age range, 44.2.3-72.4 years; 20 males and 20 females) were obtained from the China-Japan Union Hospital of Jilin University (Changchun, China) between April 2012 and April 2014. The plasma cells were purified from bone marrow aspirates using CD138 MicroBeads (cat. no. 130-051-301; Miltenyi Biotec GmbH, Bergisch Gladbach, Germany), as described previously (19). The present study was approved by the Ethics Committee of Jilin University (Changchun, China) in accordance with the Declaration of Helsinki (2000) and written informed consent was obtained from all participants.

Three MM cell lines H929, MM1S and RPMI8226, and normal plasma cells (nPCs; cat. no. PCS-800-010 ${ }^{\mathrm{TM}}$ ) were purchased from the American Type Culture Collection (Manassas, VA, USA). These cells were cultured in RPM I640 medium (Gibco; Thermo Fisher Scientific, Inc., Waltham MA USA) supplemented with $10 \%$ heat-inactivated fetal bovine serum (FBS; Gibco; Thermo Fisher Scientific Inc.), penicil in $(100 \mathrm{U} / \mathrm{ml})$ and streptomycin $(100 \mathrm{U} / \mathrm{ml})$ (Thermo Eisher Scientific, Inc.) at $37^{\circ} \mathrm{C}$ in a humidified atmosphere with $5 \%$ $\mathrm{CO}_{2}$.

Plasmid construction and transfection. miR-497 mimics, a miR-497 inhibitor, the corresponding negative control mimics (miR-NC), short hairpin (sh)RNA targeting LINC00152 (sh-LINC00152) and the empty lentiviral vector (sh-NC) were chemically synthesized and constructed by GenePharma Co., Ltd. (Shanghar, China). MM1S cells in the logarithmic growth phase were tran fected with the abovementioned products using Lipofectamine 2000 (Invitrogen; Thermo Fisher Scientific, Inc.) according to the manufacturer's instructions. After transfection for $48 \mathrm{~h}$, MM1S cells were screened with puromycin aminonucleoside to obtain those cells that stably expressed sh-LINC00152 after being transfected with the sh-LINC00152 plasmid. The transfection efficiency was assessed by reverse transcription-quantitative polymerase chain reaction (RT-qPCR) analysis.

$R T-q P C R$. Total RNA was extracted from cultured cells and tissues using a high purity total RNA extraction kit (cat no. K0801; BioTeke Co., Beijing, China) according to the manufacturer's instructions. The miR-497 expression levels were measured using the TaqMan MicroRNA assay Kit (cat no. 4366596; Thermo Fisher Scientific, Inc.) in an ABI 7900 real-time PCR system (Thermo Fisher Scientific, Inc.) according to the manufacturer's instructions. For the detection of LINC00152, complementary (c)DNAs were synthesized from RNA templates by using the RevertAid First Strand cDNA synthesis kit (Thermo Fisher Scientific, Inc.). The RT products were then amplified using SYBR Green reaction mix (Solarbio, Beijing, China) in an ABI 7900 real-time PCR system. The primers used in the present study were as follows: miR-497 sense, 5'-ACACTCCAGCTG GGCAGCAGCACACTGTGG-3' and anti-sense, 5'-TGG TGTCGTGGAGTCG-3'; U6 sense, 5'-TGCGGGTGCTCG CTTCGGCAGC-3' and anti-sense, 5'-CCAGTGCAGGGT CCGAGGT-3'. LINC00152 sense, 5'-TTGATGGCTTGA ACATTTGG-3' and anti-sense, 5'-TCGTGATTTTCGGTG TCTGT-3'; GAPDH sense, 5'-GAGTCAACGATTTGGTCG T-3' and anti-sense, 5'-GACAAGCTTCCCGTTCTCAG-3'. The following PCR conditions were used: Denaturation at $94^{\circ} \mathrm{C}$ for $3 \mathrm{~min}$, followed by 40 cycles of amplification (denaturation at $94^{\circ} \mathrm{C}$ for $10 \mathrm{sec}$, annealing at $60^{\circ} \mathrm{C}$ for $30 \mathrm{sec}$ and extension at $72^{\circ} \mathrm{C}$ for $40 \mathrm{sec}$ ). Relative quantification of the target genes was performed with the comparative quantification cycle ( $C$ q) method $\left(2 \Delta C_{4}\right)$ (21). U6 and GAPDH were assessed ats endogenous controls.

Cell proliferation and colony formation assays. Transfected cells were seeded in 96 -well plates at a density of $5 \times 10^{3}$ cells/well in RPMI-1640 medium supplemented with $10 \%$ FBS. The proliferation of the cancer cells was measured with a Cell Counting Kit-8 (CCK-8; Dojindo Laboratories, Kumamoto, following the manufacturer's protocol. The optical density (OD) at $450 \mathrm{~nm}$ was detected using a Benchmark Plus microplate spectrometer (Bio-Rad Laboratories, Hercules, CA, USA).

MM1S cells stably expressing sh-LINC00152 were seeded on 6-well plates at a density of 500 cells/well and cultured for 10 days. The medium was changed every two days. After being gently washed with PBS, the colonies were then fixed with $4 \%$ paraformaldehyde for $30 \mathrm{~min}$ at $37^{\circ} \mathrm{C}$ and stained with $0.1 \%$ crystal violet for $5 \mathrm{~min}$ at $37^{\circ} \mathrm{C}$. The visible colonies, consisting of $>50$ cells, were manually imaged and counted under a light microscope (Olympus Corp., Tokyo, Japan).

Cell cycle and apoptosis assays. For cell cycle analysis, cells were harvested via trypsinization at $48 \mathrm{~h}$ post-transfection, washed with cold PBS and fixed in 70\% ice-cold ethanol overnight, followed by staining propidium iodide (PI, $50 \mathrm{mg} / \mathrm{ml}$ ) in the presence of RNase A $(50 \mathrm{mg} / \mathrm{ml}$; Thermo Fisher Scientific, Inc.) for $30 \mathrm{~min}$ at $37^{\circ} \mathrm{C}$.

For analysis of apoptosis, cells were harvested and re-suspended in fixation fluid at $48 \mathrm{~h}$ post-transfection. Apoptosis was determined using an Annexin V-FITC/PI apoptosis detection kit (BD Biosciences, San Jose, CA, USA). Cell cycle and apoptosis were analyzed on a FACSCalibur flow cytometer (BD Biosciences) and evaluation was performed with CellQuest 3.0 software (BD Biosciences).

Caspase-3/-9 activity assay. Caspase-3/-9 activity was determined by a Caspase-3/-9 Activity Assay Kit (cat. no. AAT-22820; Beyotime Institute of Biotechnology, Beijing, China) following the manufacturer's protocol. Caspase-3/-9 activities were determined by measuring the OD at $405 \mathrm{~nm}$ using a microplate reader (BioTek Instruments, Inc., Winooski, VT, USA). 

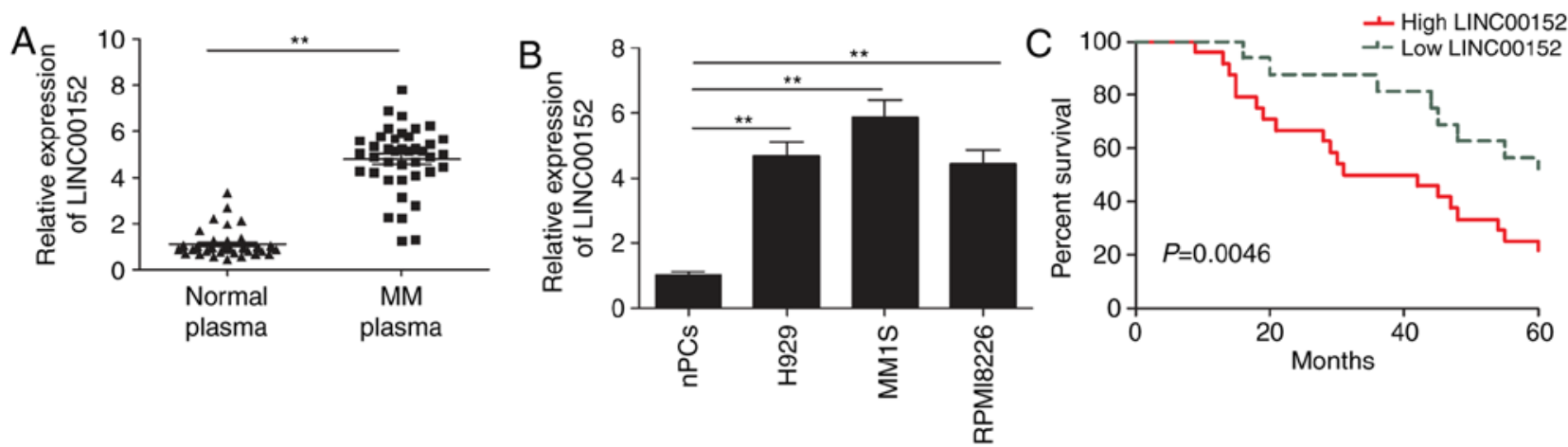

Figure 1. LINC00152 expression in MM and its association with prognosis. (A) Expression of LINC00152 in plasma cells from 40 patients with MM and 40 healthy control subjects. (B) Expression of LINC00152 in three MM cell lines and nPCs. (C) Kaplan-Meier overall survival curves for MM patients stratified by high/low LINC00152 levels. Patients with high LINC00152 expression had a reduced survival as compared with those with low LINC00152 expression. ${ }^{* *} \mathrm{P}<0.01$. MM, multiple myeloma; LINC, long non-coding RNA; mPCs, normal plasma cells.

Dual luciferase assay. The binding sites between LINC00152 and miR-497 were predicted using miRcode software (http:// www.mircode.org). Fragments of the 3' untranslated region (UTR) of LINC00152 containing the putative wild-type (WT) or mutated (MT) miR-497 binding site were chemically synthesized and cloned into the pmirGLO Dual-Luciferase miRNA Target Expression Vector (Promega Corp., Madison, WI, USA) between the XhoI and NotI sites, and named WT-LINC00152-3'UTR or MT-LINC00152 3'UTR. For the reporter assays, MM cells were co-transfected with WT-LINC00152-3'UTR or MT-LINC00152-3 TR reporter plasmid and miR-497 mimics or miR-NC using Lipofectamine 2000 (Invitrogen; Thermo Fisher Scientific, Inc.). Firefly and Renilla luciferase activities in cell lysates were measured using the Dual Luciferase Reporter Assay system (Promega Corp.) at 48 post transfection. The relative luciferase activity was standardized to the Renilla luciferase activity.

Xenograft tumor modeh A total 10 male BALB/c-nu nude mice (age, 5-6 weeks. weight, 18-20 g) were obtained from the Experimental Animal Center of Jilin University (Changchun, China). A1 animal experiments were approved by the Ethics Commite of Jilin University (Changchun, China) and complied within the Guidelines for the Welfare and Use of Animals in Cancer Research (ad hoc committee of the National Cancer Research Institute, UK).

MM1S cells stably expressing sh-LINC00152 or sh-NC were subcutaneously injected into nude mice at a dose of $2 \times 10^{6}$ cells/mouse. The tumor growth was monitored by measuring tumor length (L) and width (W) weekly and calculating the volume $(\mathrm{V})$ by using the formula $\mathrm{V}=\left(\mathrm{L} \mathrm{x} \mathrm{W}^{2}\right) / 2$. All animals were sacrificed at five weeks after tumor cell inoculation, and the subcutaneous tumors were excised and weighed. The tumor tissues were stored at $-80^{\circ} \mathrm{C}$ until they were used to detect LINC00152 or miR-497 expression by RT-qPCR. Other parts of the tumors were fixed in neutral formalin, dehydrated and embedded in paraffin for further analysis.

Immunohistochemistry (IHC). IHC was performed on paraffin-embedded xenograft tumors as described previously (20). Primary antibodies to Ki-67 were purchased from Santa Cruz Biotechnology, Inc. (Dallas, TX, USA; cat. no. 23900 ) and used at a 1:400 dilution.

Statistical analysis. Values are expressed as the mean \pm standard deviation from at least three independent repeats of the experiments. Al statistical analyses were performed using SPSS r. 19.0 (IBM Corp., Armonk, NY, USA). Student's t-test was used for comparisons between two groups. One-way analysis of variance with Tukey's post-hoc test was employed to estimate the significant differences if $>2$ groups were present. Spearman's correlation analysis was used to analyze correlation in a data-set. The survival time of the patients was analyzed using the Kaplan-Meier method and the log-rank test. $\mathrm{P}<0.05$ was considered to indicate a statistically significant difference between the groups.

\section{Results}

LINC00152 is upregulated in plasma cells from MM patients and MM cell lines. First, the expression levels of LIN00152 in plasma cells from $40 \mathrm{MM}$ patients and 40 healthy donors were examined. RT-qPCR demonstrated that the expression of LINC00152 was significantly higher in plasma cells from MM patients than in those from healthy donors (Fig. 1A). Subsequently, the expression of LINC00152 in the three MM cell lines H929, MM1S and RPMI8226, and in the nPCs was evaluated. As presented in Fig. 1B, LINC00152 was significantly upregulated in the three MM cell lines as compared with that in nPCs $(\mathrm{P}<0.01)$. These results suggest that LINC00152 may have a role in the genesis of MM. Next, the MM patients were divided into two groups according to the expression levels of LINC00152 by using the median LINC00152 expression value as a cut-off, and it was further assessed whether the expression of LINC00152 is associated with the survival time of the patients using Kaplan-Meier analysis and the log-rank test. The results indicated that high LINC00152 expression corresponded with a significantly shorter overall survival as compared with that in the low expression group (Fig. 1C).

LINC00152 knockdown inhibits MM cell proliferation and induces cell cycle arrest. To assess the effect of LINC00152 
A

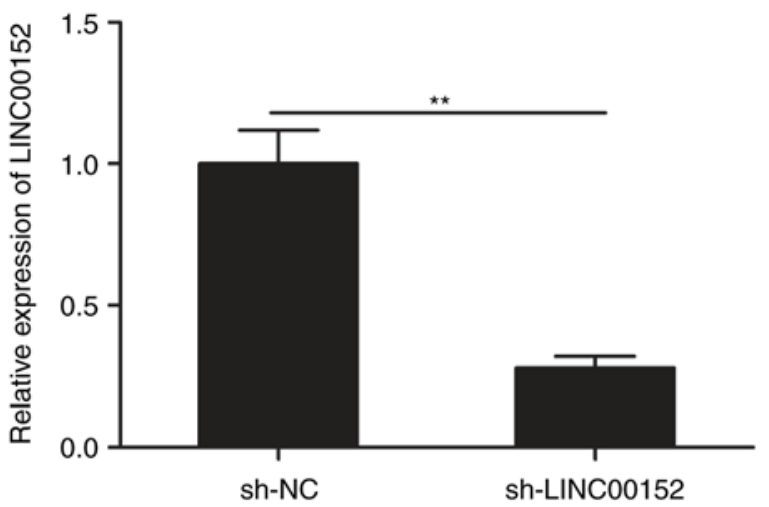

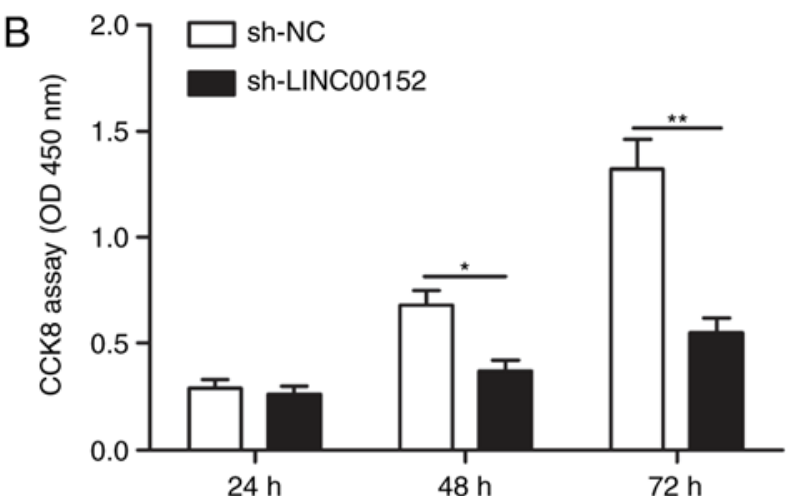

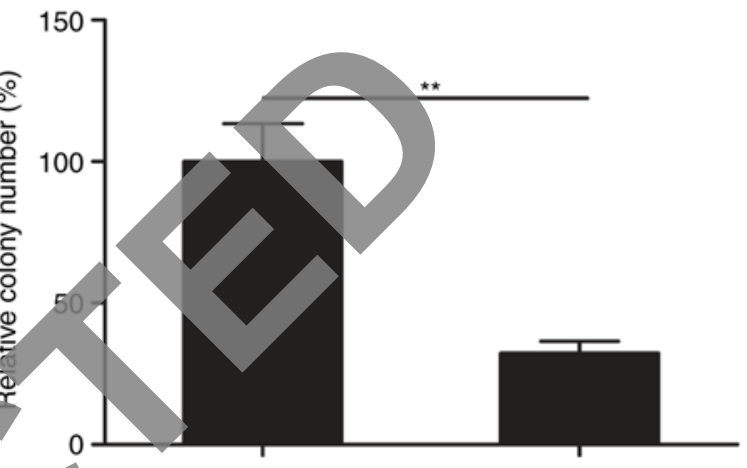

sh-NC sh-LINC00152

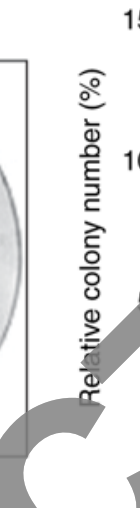

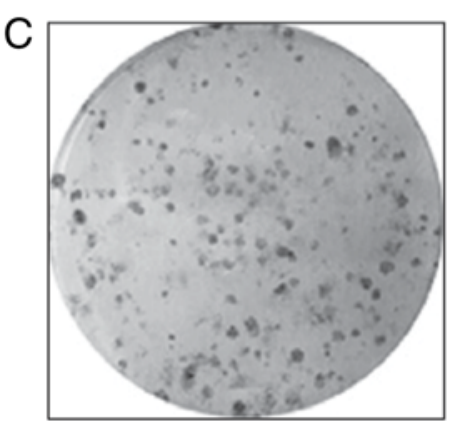

sh-NC

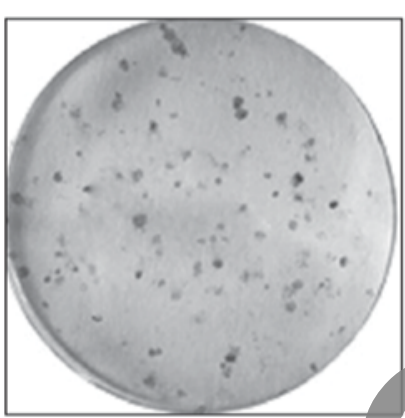

sh-LINC00152

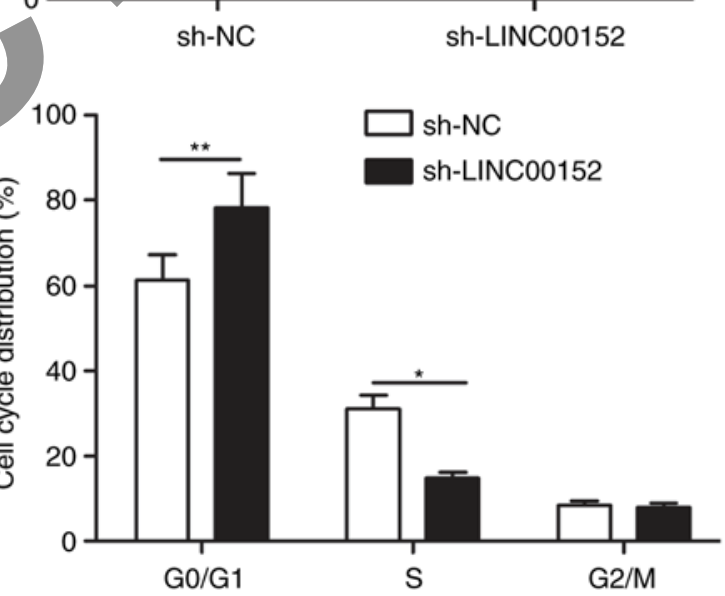

Figure 2. LINC00152 knockdown in hibits MM cell proliferation and induces cell cycle arrest. (A) LINC00152 expression levels were detected in MM1S cells transfected with S-LINC00152 sh-NC plasmids. Subsequently, (B) cell proliferation, (C) colony formation (magnification, x100) and (D) cell cycle distribution were determined in MM1S cells transfected with sh-LINC00152 or sh-NC plasmids. ${ }^{*} \mathrm{P}<0.05$, ${ }^{* *} \mathrm{P}<0.01$. sh-NC, negative control small hairpin RNA; sh-LINC00152, shRNA targeting long non-coding RNA 00152; OD, optical density; CCK-8, Cell Counting Kit-8; 2N, G0/G1 stage; 4N, G2/M stage.

on MM cells, the LINC00152 gene was knocked down in MM1S cells using sh-LINC00152 plasmid. The transfection efficiency was verified by RT-qPCR, revealing that MM1S cells transfected with the sh-LINC00152 plasmid had a significantly decreased LINC00152 expression as compared with that in sh-NC-transfected cells (Fig. 2A). The CCK-8 assay indicated that knockdown of LINC00152 in MM1S cells significantly reduced their viability (Fig. 2B) and furthermore, their colony formation ability was significantly impaired (Fig. 2C). To investigate the mechanisms involved in the effect of LINC00152 on the proliferation and clonogenicity of MM cells, the cell cycle was assessed using flow cytometry. The results demonstrated that LINC00152 knockdown induced cell cycle arrest of $M M$ cells at $\mathrm{G}_{0} / \mathrm{G}_{1}$ phase and decreased cell cycle progression to the $\mathrm{S}$ phase (Fig. 2D).
LINC00152 knockdown induces MM-cell apoptosis. The effect of LINC00152 silencing on the apoptosis of MM1S cells was then explored by fluorescence-assisted cell sorting analysis. As presented in Fig. 3A, LINC00152 knockdown significantly induced apoptosis in MM1S cells $(\mathrm{P}<0.01)$. In order to investigate the underlying mechanism, the activities of caspase- 3 and caspase- 9 were measured with a Caspase-3/-9 Activity Assay Kit. The intracellular activities of caspase-3 and caspase-9 were significantly increased in MM1S cells transfected with sh-LINC00152 as compared with that in sh-NC-transfected cells (Fig. 3B and C).

miR-497 expression is directly regulated by LINC00152. Vast evidence has indicated that lncRNAs function as competing endogenous RNAs (ceRNAs) by competitively binding to 

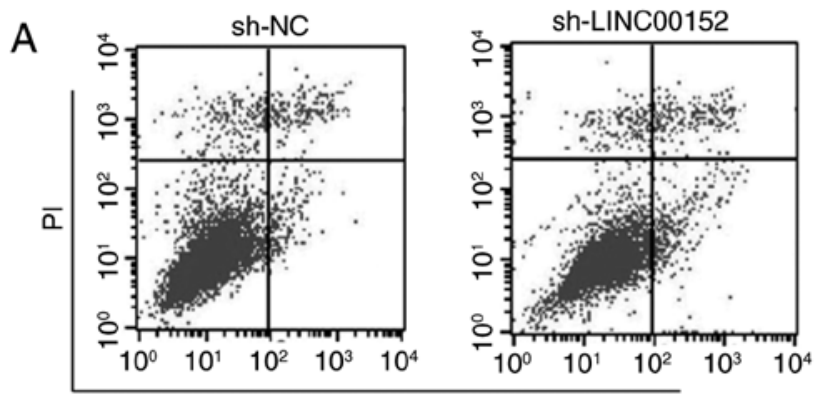

Annexin V FITC
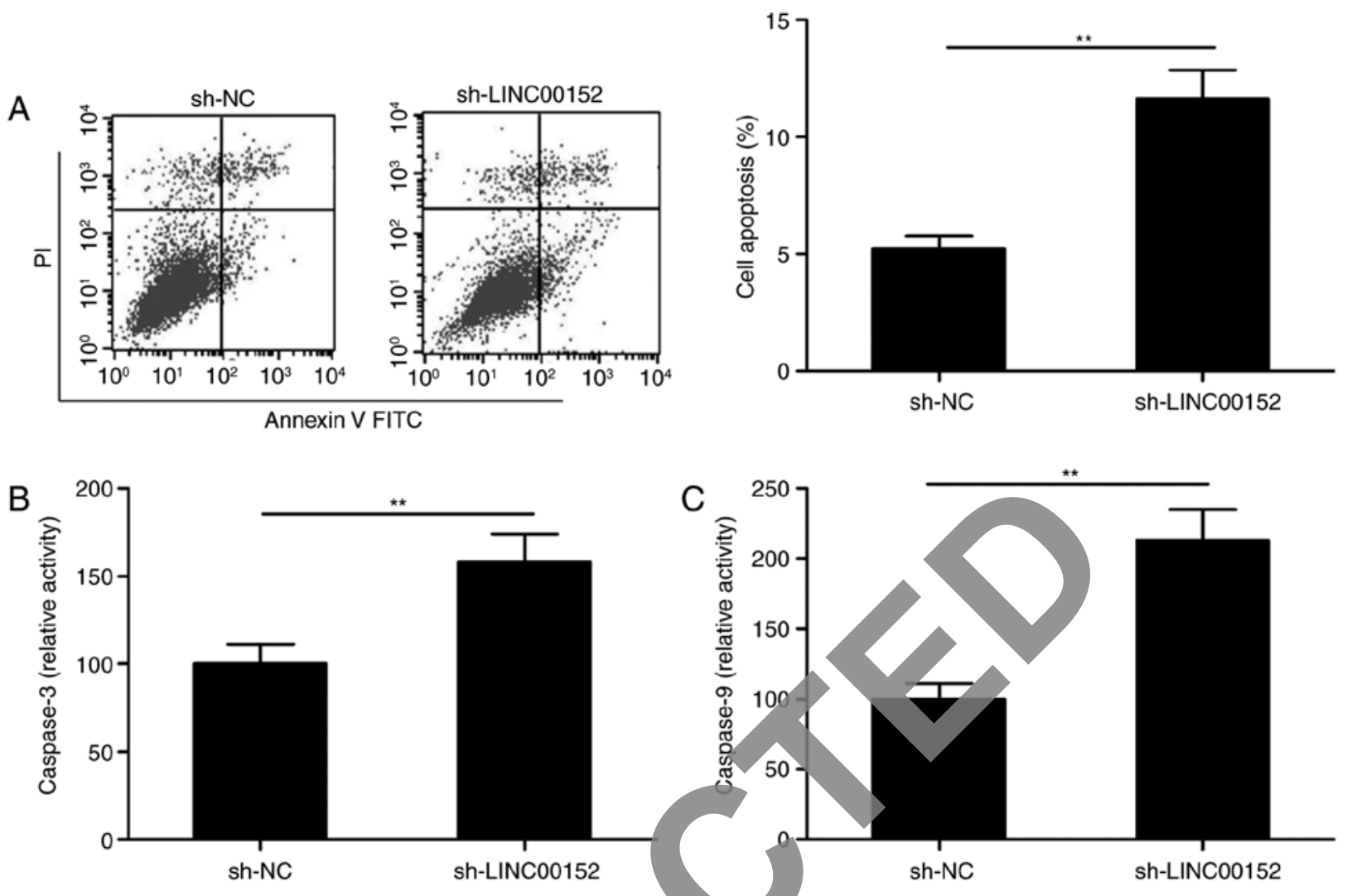

Figure 3. LINC00152 knockdown induces MM cell apoptosis. (A) Cell apoptosis was determined in MM1S cells transfected with sh-LINC00152 or sh-NC plasmids. (B) Caspase-3 and (C) caspase-9 activities were determine in MM1S cells transfected with sh-LINC00152 or sh-NC plasmids. ${ }^{* *} \mathrm{P}<0.01$. sh-NC, negative control small hairpin RNA; sh-LINC00152, shRNA targeting lons non-coding RNA 00152; PI, propidium iodide; FITC, fluorescein isothiocyanate.
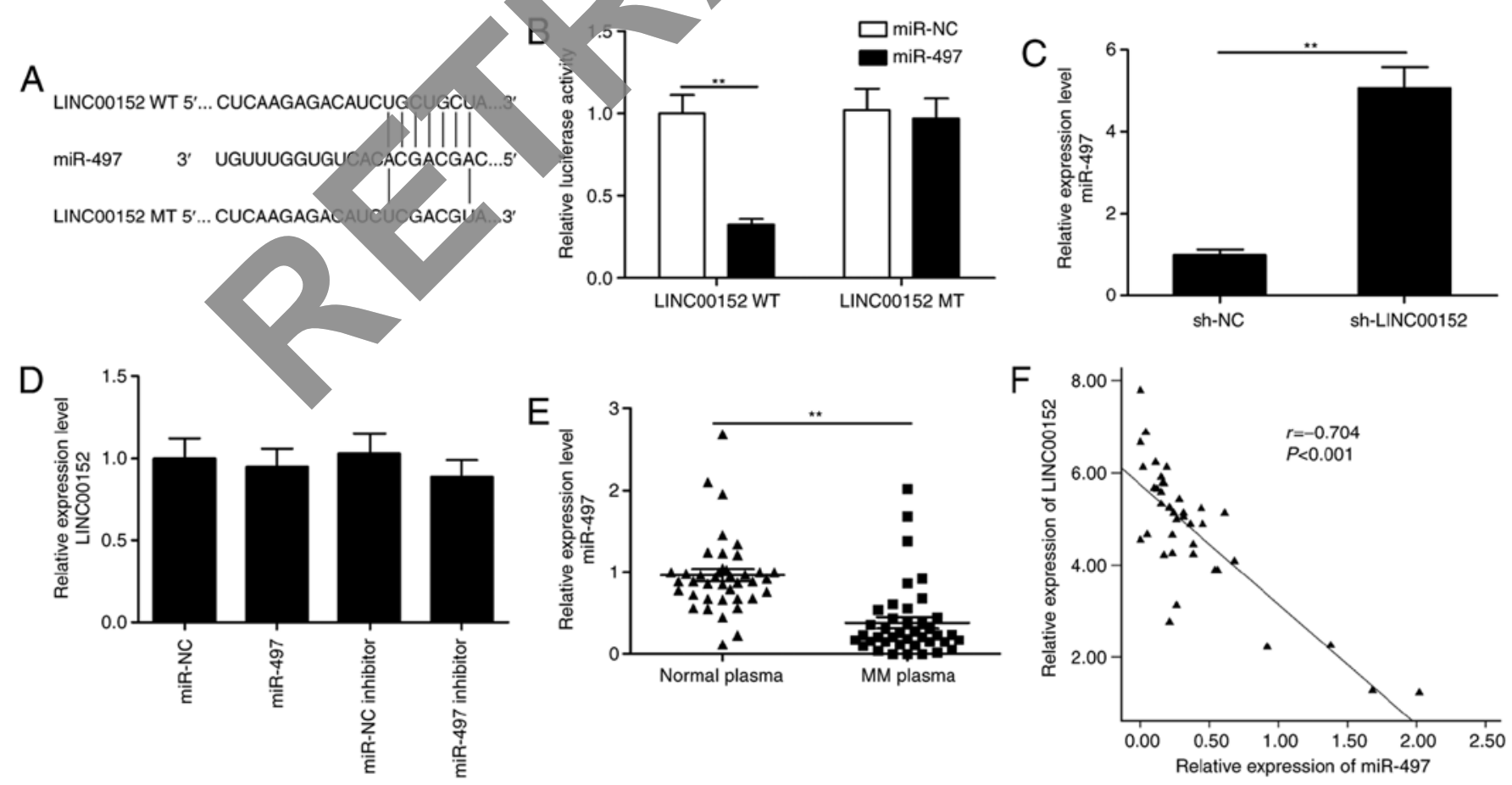

Figure 4. miR-497 expression is directly regulated by LINC00152. (A) Schematic representation of the predicted binding sites between miR-497 and LINC00152, and the mutagenesis design for the reporter assays. (B) Luciferase activity in MM1S cells co-transfected with miR-497 mimics or miR-NC, and luciferase reporters containing LINC00152-WT or LINC00152-MT. (C) miR-497 expression levels were determined in MM1S cells transfected with sh-LINC00152 or sh-NC plasmids. (D) LINC00152 expression levels were determined in MM1S cells transfected with miR-497 mimics or miR-497 inhibitor. (E) Expression of miR-497 in plasma cells from 40 patients with MM and 40 healthy control subjects. (F) An inverse association between LINC00152 and miR-497 expression in plasma from MM patients $(n=40)$ was identified by Spearman's correlation analysis. ${ }^{* *} \mathrm{P}<0.01$. sh-NC, negative control small hairpin RNA; sh-LINC00152, shRNA targeting long non-coding RNA 00152; miR, microRNA; WT, wild-type; MT, mutant; MM, multiple myeloma. 

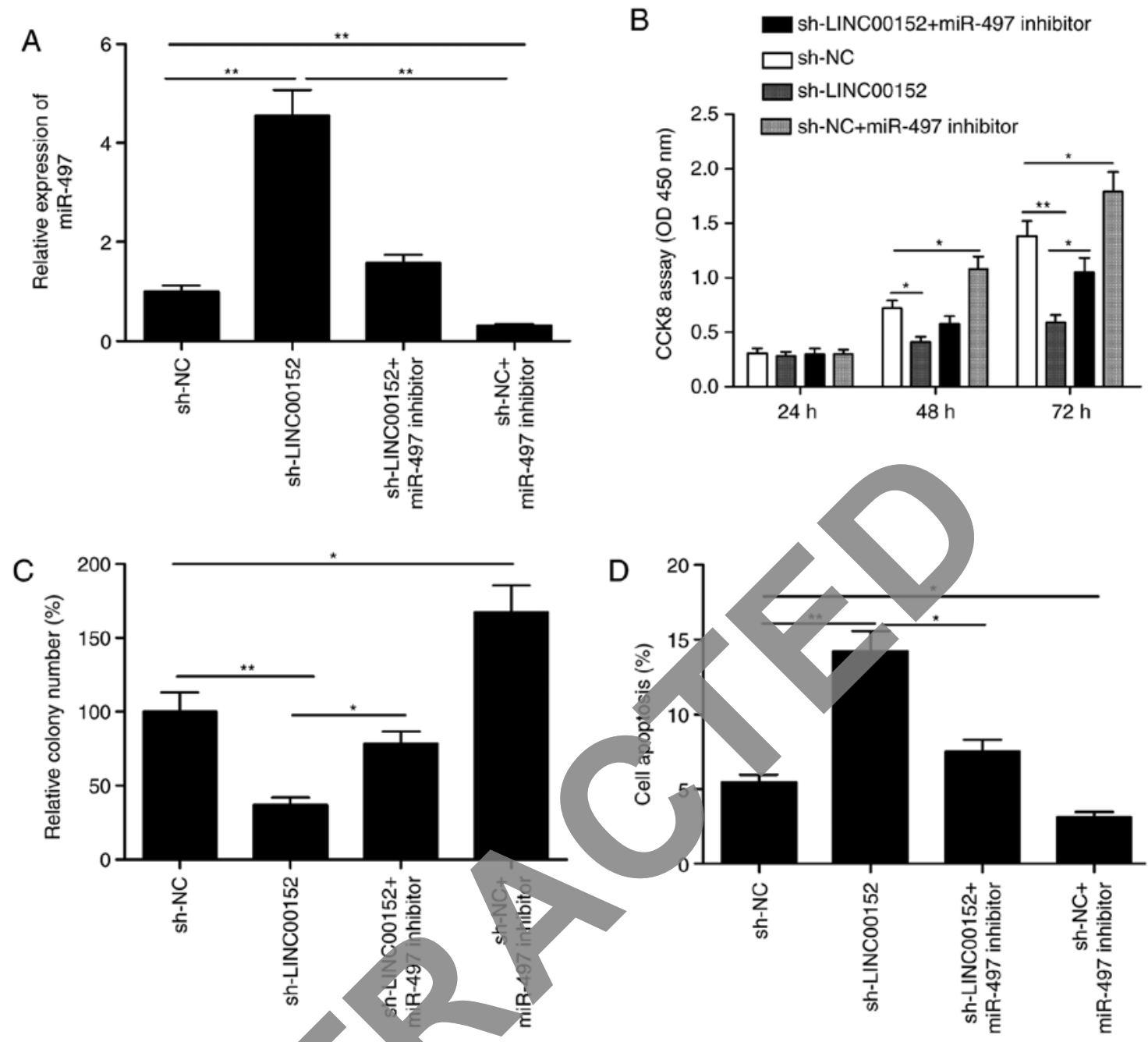

Figure 5. Repression of miR-497 inversed the sh-LINC00152-Induced inhibitory effects on MM cells. (A) miR-497 expression was determined in MM1S cells transfected with sh-LINC00152 or sh-NC, with or without the miR-497 inhibitor. (B) Cell proliferation, (C) colony formation and (D) apoptosis were determined in MM1S cells transfected with sh-LINC00152 or sh-NC, with or without the miR-497 inhibitor. "P<0.05, ${ }^{* * *} \mathrm{P}<0.01$. miR, microRNA; sh-NC, negative control small halrpin RNA; sh-LING.00152, shRNA targeting long non-coding RNA 00152; OD, optical density; CCK-8, Cell Counting Kit-8.

miRNAs. To identily miRNAs that are directly targeted by LINC00152 in MM cels, the bioinformatics tool miRcode was used to predict potential target miRs of LINC00152, of which miR-497 was selected as a putative target (Fig. 4A). To confirm the direct binding interaction between LINC00152 and miR-497, a luciferase reporter assay was performed. The results demonstrated that miR-497 mimics reduced the luciferase activity of LINC00152-WT but not of LINC00152-MT vectors (Fig. 4B; P<0.05). Furthermore, LINC00152 knockdown significantly increased the levels of miR-497 in MM cells (Fig. 4C; P<0.05). However, miR-497 mimics or inhibitor did not affect LINC00152 levels in MM cells (Fig. 4D; P>0.05). In addition, in plasma cells from MM patients, the expression of miR-497 was significantly downregulated and was inversely correlated with the expression of LINC00152 (Fig. 4E and F). These results indicate that LINC00152 directly binds to miR-497 to reduce its availability.

Repression of miR-497 restores the inhibitory effects of sh-LINC00152 on MM cells. To investigate whether LINC00152 exerts its oncogenic effect on MM cells through
miR-497, a loss-of-function experiment was performed by inhibiting miR-497 expression in LINC00152-knockdown MM1S cells (Fig. 5A). The CCK-8 assay and the colony formation assay indicated that the miR-497 inhibitor promoted the proliferation and colony formation (Fig. $5 \mathrm{~B}$ and $\mathrm{C} ; \mathrm{P}<0.05$ ), and partially abrogated the LINC00152 knockdown-induced reduction in cell proliferation and colony formation ability on MM1S cells (Fig. 5B and C; $\mathrm{P}<0.05$ ). Flow cytometric analysis further indicated that apoptosis was increased in LINC00152-knockdown MM1S cells, which was partially abrogated by co-transfection with miR-497 inhibitor (Fig. 5D; $\mathrm{P}<0.05)$. These results suggest that repression of $\mathrm{miR}-497$ partially restored the inhibitory effects of sh-LINC00152 on MM cells.

LINC00152 knockdown suppresses tumor growth in vivo. To further investigate the role of LINC00152 in tumor growth in vivo, MM1S cells stably expressing sh-LINC00152 or sh-NC were subcutaneously injected into nude mice. Xenograft tumors were evaluated over the duration of the experiment and excised at seven weeks after inoculation. It was indicated 

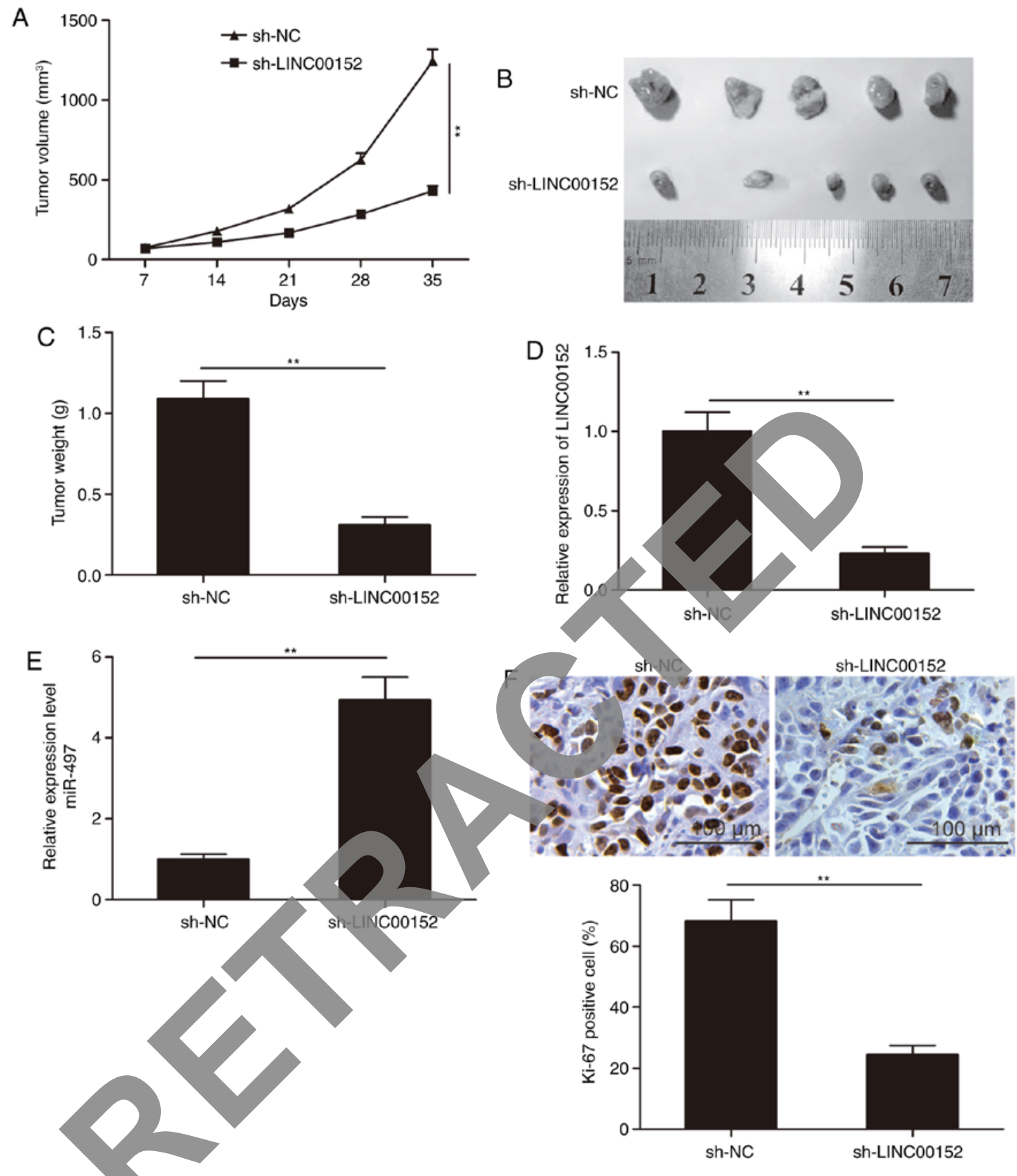

Figure 6. LINC00152 knockdown suppresses tumor growth in vivo. (A) The tumor volume was calculated every seven days after inoculation of multiple myeloma cells. (B) Images and (C) weight of the excised tumors. (D and E) The expression levels of (D) LINC00152 and (E) miR-497 in the tumor tissues were determined. (F) Immunohistochemical analysis of Ki-67 expression in the subcutaneous tumors of mice (scale bar, $100 \mu \mathrm{m}$ ). ${ }^{* *} \mathrm{P}<0.01$. sh-NC, negative control small hairpin RNA; sh-LINC00152, shRNA targeting long non-coding RNA 00152; miR, microRNA.

that the tumor volumes and final weight in the sh-LINC00152 group were significantly lower than those in the sh-NC group $(\mathrm{P}<0.05$; Fig. 6A-C). Furthermore, LINC00152 and miR-497 expression in tumor tissues was examined by RT-qPCR. The results indicated that LINC00152 expression was significantly decreased, whereas miR-497 expression was increased in the sh-LINC00152 group as compared with that in the sh-NC group $(\mathrm{P}<0.05$; Fig. $6 \mathrm{D}$ and $\mathrm{E})$. IHC staining of the tumors revealed that the number of $\mathrm{Ki}-67$-positive cells was obviously reduced in the sh-LINC00152 group as compared with that in the sh-NC group (Fig. 6F). These results suggest that LINC00152 knockdown reduces the tumorigenesis of MM in vivo.

\section{Discussion}

Increasing evidence has suggested that dysregulation of IncRNAs is involved in the genesis and progression of MM $(10,11)$. For instance, overexpression of lncRNA imprinted RNA near maternally expressed 3 promoted the osteogenic differentiation of mesenchymal stem cells from MM patients by targeting the transcription of bone morphogenetic protein 4 (22). Knockdown of the IncRNA H19, imprinted maternally expressed transcript by shRNA transfection significantly inhibited the proliferation, viability and colony formation in $\mathrm{MM}$ cells, and inactivated the nuclear factor- $\mathrm{\kappa B}$ pathway (23). Knockdown of lncRNA metastasis-associated lung 
adenocarcinoma transcript 1 significantly suppressed the cell proliferation, induced apoptosis, caused cell cycle arrest in $\mathrm{G}_{1} / \mathrm{S}$ phase and inhibited MM cell growth in vivo through sponging miR-509-5p to modulate forkhead box P1 expression (24). lncRNA colon cancer-associated transcript 1 promoted MM growth in vitro and in vivo by acting as a molecular sponge for miR-181a-5p to modulate homeobox A1 expression (25). In the present study, it was identified that LINC00152 was highly expressed in plasma cells from MM patients and MM cell lines, and the upregulation of LINC00152 in their plasma cells was significantly associated with poor prognosis of MM patients. In addition, it was verified that LINC00152 knockdown significantly suppressed MM cell proliferation and clonogenicity, and induced apoptosis. These results suggest that LINC00152 may serve as a promising target for therapeutic intervention in MM.

Several studies have demonstrated that LINC00152 has considerable functional roles in various human malignancies by regulating cancer cell proliferation, apoptosis, metastasis, migration and invasion (12-20). It was reported to be upregulated and to function as an oncogene in various cancer types $(26,27)$. However, the role of LINC00152 in MM and the underlying mechanisms have remained largely elusive. In the present study, RT-qPCR confirmed that LINC00152 was highly expressed in plasma cells from MM patients and MM cell lines, and that high LINC00152 expression in plasma cells was associated with a relatively shorter survival of patients with MM. In addition, knockdown of LINC00152 in MM1S cells significantly suppressed their proliferation and colony formation, while promoting cell apoptosis, as well as and caspase-3 and caspase -9 activity. An in vivo xenograft experiment demonstrated that knockdown of LINC00152 suppressed the grow th of tumors derived from MM1S cells in nude mice These restlts suggest that LINC00152 functions as an oncogene in human MM.

lncRNAs are known to be involved in a series of cellular biological processes by acting as ceRNAs or molecular sponges to modulate the effect of miRNAs $(28,29)$. Previous studies have demonstrated that LINC0 152 has an oncogenic role in colorectal cancer by regu ating miR $376 c-3 p$ (30), in glioma by regulating miR-103a-3p $(31)$ and in gastric cancer by targeting miR-139-5p (32). In the present study, miR-497 was identified as an inhibitory target of LINC00152 by sequence complementarity analysis and luciferase reporter assays. miR-497, a member of the miR-15 family, has been reported to have a critical role in cancer progression and development (33). A study has indicated that miR-497 expression was downregulated in MM samples and cell lines, and that its overexpression significantly inhibited malignant progression of MM by directly targeting PBX homeobox 3 (34). In line with this, the present results indicated that miR-497 was downregulated in MM samples. In addition, miR-497 expression was inversely correlated with LINC00152 expression. Furthermore, LINC00152 knockdown increased miR-497 expression in MM cells. In addition, it was demonstrated that the miR-497 inhibitor partly abrogated the effect of LINC00152 knockdown on MM growth. These results strongly suggest that LINC00152 directly targets miR-497 and affects the biological characteristics of MM cells by negatively regulating miR-497.

Various limitations of the present study should be considered. First, only one MM cell line was used to examine the biological function of LINC00152 in MM progression, and experiments using further cells lines may be required for further validation. In addition, a gain-of-function study with upregulation of LINC00152 in MM cells should be performed to fully determine the role of LINC00152 in MM. Furthermore, LINC00152 may affect drug resistance of patients with MM, which requires further study.

In conclusion, the present study indicated that LINC00152 is highly expressed in plasma cells from MM patients and MM cell lines. In addition, it was revealed that LINC00152 acts as an oncogene and modulates MM cell growth by targeting miR-497. These results provided insight into the biological and clinical significance of LINC00152. Based on the present results, LINC00152 may potentially be utilized as a biomarker for MM and may serve as a therapeutic target for the treatment of patients with MM

\section{Acknowledgements \\ Not applicable. \\ Fun \\ No runding was received. \\ Availability of data and materials}

The analyzed datasets generated during the study are available fom the corresponding author on reasonable request.

\section{Authors' contributions}

TY, ZX and HN conceived the study; XZ and LM performed the experiments; TY and ZX analyzed the data; and TY, ZX and $\mathrm{HN}$ wrote the manuscript. All authors read and approved the final manuscript.

\section{Ethics approval and consent to participate}

The present study was approved by the Ethics Committee of Jilin University (Changchun, China) in accordance with the Declaration of Helsinki (2000) and written informed consent was obtained from all participants.

\section{Patient consent for publication}

Not applicable.

\section{Competing interests}

The authors declare that they have no competing interests.

\section{References}

1. Torre LA, Bray F, Siegel RL, Ferlay J, Lortet-Tieulent J and Jemal A: Global cancer statistics, 2012. CA Cancer J Clin 65: 87-108, 2015.

2. Alexander DD, Mink PJ, Adami HO, Cole P, Mandel JS Oken MM and Trichopoulos D: Multiple myeloma: A review of the epidemiologic literature. Int J Cancer 12(Suppl 120): S40-S61, 2007. 
3. Hideshima T, Richardson PG and Anderson KC: Mechanism of action of proteasome inhibitors and deacetylase inhibitors and the biological basis of synergy in multiple myeloma. Mol Cancer Ther 10: 2034-2042, 2011.

4. Kornienko AE, Guenzl PM, Barlow DP and Pauler FM: Gene regulation by the act of long non-coding RNA transcription. BMC Biol 11: 59, 2013.

5. Ponting CP, Oliver PL and Reik W: Evolution and functions of long noncoding RNAs. Cell 136: 629-641, 2009.

6. Mercer TR, Dinger ME and Mattick JS: Long non-coding RNAs: Insights into functions. Nat Rev Genet 10: 155-159, 2009.

7. Geisler $S$ and Coller J: RNA in unexpected places: Long non-coding RNA functions in diverse cellular contexts. Nat Rev Mol Cell Biol 14: 699-712, 2013.

8. Sun T: Long noncoding RNAs act as regulators of autophagy in cancer. Pharmacol Res 129: 151-155, 2018.

9. Fan Q, Yang L, Zhang X, Peng X, Wei S, Su D, Zhai Z, Hua X and $\mathrm{Li} \mathrm{H}$ : The emerging role of exosome-derived non-coding RNAs in cancer biology. Cancer Lett 414: 107-115, 2018.

10. Hu AX, Huang ZY, Zhang L and Shen J: Potential prognostic long non-coding RNA identification and their validation in predicting survival of patients with multiple myeloma. Tumour Biol 39: 1010428317694563, 2017.

11. Ronchetti D, Manzoni M, Todoerti K, Neri A and Agnelli L: In silico characterization of miRNA and long non-coding RNA interplay in multiple myeloma. Genes (Basel) 7: E107, 2016.

12. Liu L, Wen J, Gu X, Wu D, Lu M and Zhao Q: Prognostic role of long non-coding RNA LINC00152 in Chinese cancer patients: A meta-analysis. Oncotarget 8: 93227-93235, 2017.

13. Bian Z, Zhang J, Li M, Feng Y, Yao S, Song M, Qi X, Fei B, Yin Y, Hua D and Huang Z: Long non-coding RNA LINC00152 promotes cell proliferation, metastasis, and confers 5-FU resistance in colorectal cancer by inhibiting miR-139-5p. Oncogenesis 6: 395, 2017.

14. Wang X, Yu H, Sun W, Kong J, Zhang L, Tang J, Wang J, Xu E, Lai $\mathrm{M}$ and Zhang $\mathrm{H}$ : The long non-coding RNA CYTC colorectal cancer progression by interacting with $\mathrm{N}$ Sam68. Mol Cancer 17: 110, 2018.

15. Yue B, Liu C, Sun H, Liu M, Song C, Cui R, Qiu S and Zho A positive feed-forward loop between LncR A-CYTOR and Wnt $/ \beta$-catenin signaling promotes metastasis of colon cancer. Mol Ther 26: 1287-1298, 2018.

16. Wu J, Shuang Z, Zhao J, Tang H, Li P, Zhang L, Xie X and Xiao X: Linc00152 promotes tumorigenesis by regulating DNMTs in triple-negative breast cancer. Biomed Pharmacother 97 $1275-1281,2018$.

17. Zhang Y, Xiang C, Wang Y, Duan Y, Liu C, Jin Y and Zhang Y: IncRNA LINC00152 knoekdow had effects to suppress biological activity of lung cancer ya EGER/PI3K/AKT pathway. Biomed Pharmacother 94:

18. Yu J, Liu Y, Guo C, Zhang S, Gong Z, Tang Y, Yang L, He Y, Lian Y, Li X elal: Unregulated long non-coding RNA LINC00152 expression is associated with progression and poor prognosis of tongue squamoús cell carcinoma. J Cancer 8: 523-530, 2017.

19. Cai Q, Wang Z, Wang S, Weng M, Zhou D, Li C, Wang J, Chen E and Quan Z: Long non-coding RNA LINC00152 promotes gallbladder cancer metastasis and epithelial-mesenchymal transition by regulating HIF-1alpha via miR-138. Open Biol 7: 160247, 2017.
20. Wu Y, Tan C, Weng WW, Deng Y, Zhang QY, Yang XQ, Gan HL, Wang T, Zhang PP, Xu MD, et al: Long non-coding RNA Linc00152 is a positive prognostic factor for and demonstrates malignant biological behavior in clear cell renal cell carcinoma. Am J Cancer Res 6: 285-299, 2016.

21. Livak KJ and Schmittgen TD: Analysis of relative gene expression data using real-time quantitative PCR and the 2(-Delta Delta C(T)) method. Methods 25: 402-408, 2001

22. Zhuang W, Ge X, Yang S, Huang $M$, Zhuang W, Chen $P$, Zhang X, Fu J, Qu J and Li B: Upregulation of IncRNA MEG3 promotes osteogenic differentiation of mesenchymal stem cells from multiple myeloma patients by targeting BMP4 transcription. Stem Cells 33: 1985-1997, 2015.

23. Sun Y, Pan J, Zhang N, Wei W, Yu S and Ai L: Knockdown of long non-coding RNA H19 inhibits multiple myeloma cell growth via NF- $\kappa \mathrm{B}$ pathway. Sci Rep 7: 18079, 2017.

24. Gu Y, Xiao X and Yang S: LncRNA MALAT1 acts as an oncogene in multiple myeloma through sponging miR-509-5p to modulate FOXP1 expression. Oncotarget 8: 101984-101993, 2017.

25. Chen $\mathrm{L}, \mathrm{Hu} \mathrm{N}$, Wang $C$, Zhao $\mathrm{H}$ and $\mathrm{Gu} \mathrm{Y}$ : Long non-coding RNA CCAT1 promotes multiple myeloma progression by acting as a molecular sponge of miR-181a-5p to modulate HOXA1 expression. Cell Cycle 17: 319-329, 2018.

26. Ji J, Tang $J$, Deng I, Xie Y, Jiang R, Li G and Sun B: LINC00152 promotes proliferation in hepatocellular carcinoma by targeting EpCAM via the mTOR signaling pathway. Oncotarget 6: $42813-42824,20$

27. Quan FY, Jiang J, Zhai YF, Li B, Wu XH and Nie W: The prognostic effect of LINC00152 for cancer: A meta-analysis. Oncotarget 8: 75427-75433, 2017.

28. Huang C, Yuan N, Wu L, Wang X, Dai J, Song P, Li F, Xu C and Zhao X: An integrated analysis for long noncoding RNAs and microRNAs with the mediated competing endogenous RNA net ork in papillary renal cell carcinoma. Onco Targets Ther 10 : 37-4050, 2017.

29. Xue M, Zhuo Y and Shan B: MicroRNAs, long noncoding RNAs, and their functions in human disease. Methods Mol Biol 1617: 1-25, 2017.

30. Zhang YH,Fu J,Zhang ZJ, Ge CC and Yi Y: LncRNA-LINC00152 down-regulated by miR-376c-3p restricts viability and promotes apoptosis of colorectal cancer cells. Am J Transl Res 8: 5286-5297, 2016.

31. Yu M, Xue Y, Zheng J, Liu X, Yu H, Liu L, Li Z and Liu Y: Linc00152 promotes malignant progression of glioma stem cells by regulating miR-103a-3p/FEZF1/CDC25A pathway. Mol Cancer 16: 110, 2017.

32. Sun K, Hu P and Xu F: LINC00152/miR-139-5p regulates gastric cancer cell aerobic glycolysis by targeting PRKAA1. Biomed Pharmacother 97: 1296-1302, 2018.

33. Yang G, Xiong G, Cao Z, Zheng S, You L, Zhang T and Zhao Y: miR-497 expression, function and clinical application in cancer. Oncotarget 7: 55900-55911, 2016.

34. Yu T, Zhang X, Zhang L, Wang Y, Pan H, Xu Z and Pang X: MicroRNA-497 suppresses cell proliferation and induces apoptosis through targeting PBX3 in human multiple myeloma. Am J Cancer Res 6: 2880-2889, 2016. 